\title{
Periodic integration in quarterly UK macroeconomic variables
}

\author{
Philip Hans Franses* \\ Econometric Institute, Erasmus University, Rotterdam, P.O. Box 1738, NL-3000 DR Rotterdam, \\ Netherlands \\ Gerbert Romijn \\ Department of Macroeconomics, Erasmus University, Rotterdam, P.O. Box 1738, \\ NL-3000 DR Rotterdam, Netherlands
}

\begin{abstract}
This paper presents empirical evidence on the seasonal patterns in several UK macroeconomic variables, additional to related evidence reported in Osborn (International Journal of Forecasting (1990), 6, 327-336). The method used is a test procedure for seasonal unit roots that allows parameters to vary over the seasons. This extension of currently applied procedures can select between seasonal and periodic integration. In a small Monte Carlo experiment, this new method is evaluated with respect to two rival procedures. The empirical results for the UK variables indicate that many of these are periodically integrated. The implications of this outcome on modelling and forecasting are discussed. One of the implications is that a periodic error correction model for the univariate series can outperform non-periodic models with respect to forecasting.
\end{abstract}

Keywords: Seasonality; Periodic processes; Unit roots; Forecasting

\section{Introduction and summary}

Recently, Osborn (1990) has carried out a survey of seasonality in 30 UK macroeconomic variables. The methods tested, e.g. seasonal unit roots, are those developed in Hylleberg et al. (1990) [HEGY] and in Osborn et al. (1988) [OCSB]. A common characteristic of these methods is the assumption that the parameters do not vary over the seasons, i.e. that the time series can be described by non-periodic models. However, since there is evidence that periodic models can have an improved forecasting performance over non-periodic models [see, for example, Osborn and Smith (1989)], it seems worthwhile to consider methods for the investigation of seasonal patterns that include periodic processes. The purpose of the present paper is to survey the evidence on seasonality in UK macroeconomic variables to be obtained by applying a periodic extension of the HEGY method. We further evaluate, via a limited simulation exercise, the effects that periodic data-generating processes have on the outcomes of the two non-periodic test methods.

* Corresponding author. 
In section 2 we briefly review the three methods. Detailed accounts of their properties can be found in the corresponding references. In section 3, a limited simulation exercise is carried out. In section 4 we report on the results on UK macroeconomic time series. It turns out that many quarterly UK macroeconomic time series are periodically integrated, i.e. the time series are non-stationary and have time-varying parameters. In section 5 we discuss some of the implications of this outcome for modelling and forecasting. One conclusion is that an improvement in forecasting performance can be observed in the case where a univariate periodic error correction model is used. An extension to a periodic cointegration model is briefly discussed. In section 6 we conclude with some remarks.

\section{A review of methods}

Consider a quarterly observed time series, $X_{t}$, where $t=1, \ldots, n$. Many economic time series appear to be non-stationary, or integrated to some order, to be denoted as $I(\cdot)$. For quarterly time series the most often encountered cases are of type $I(d, D)$, where $d$ and $D$ are either 0 or 1 . A series is said to be $I(d, D)$ when the filter $\Delta_{1}^{d} \Delta_{4}^{D}$ is required for stationarity, where a filter is defined by $\Delta_{k}^{m} y_{t}=\left(1-B^{k}\right)^{m} y_{t}=\left(1-B^{k}\right)^{m-1}\left(y_{t}-y_{t-k}\right)$, with $\Delta_{k}^{0}=1$. For example, a series that is $I(1,0)$ requires the use of the $\Delta_{1}$ filter to make the series stationary.

One method of selecting between these filters for a non-stationary seasonal time series is proposed in Osborn et al. (1988). It amounts to estimating the auxiliary regression

$$
\Delta_{1} \Delta_{4} X_{t}=\sum_{s=1}^{4} \alpha_{s} D_{s t}+\beta_{1} \Delta_{1} X_{t-1}+\beta_{2} \Delta_{1} X_{t-4}+\sum_{i=1}^{p} \phi_{i} \Delta_{1} \Delta_{4} X_{t}{ }_{i}+u_{t},
$$

where each $D_{s t}$ is a seasonal dummy variable. It can be derived that decisions on the order of integration are related to the significance of $\beta_{1}$ and $\beta_{2}$. When $\beta_{1}=\beta_{2}=0$, the series $X_{1}$ is $I(1,1)$, and when they are both unequal to zero, the series is stationary, i.e. $I(0,0)$. When $\beta_{1}<0$ and $\beta_{2}=0$, the series is $I(0,1)$, and when $\beta_{1}=0$ while $\beta_{2}<0$, the series is $I(1,0)$. See Osborn (1990) for additional details and for critical values of several test statistics.

The method to test for seasonal unit roots developed in HEGY considers the selection between $I(0,0), I(0,1), I(1,0)$ and some intermediate cases which are characterized by the fact that $\left(1-B^{4}\right)$ equals $(1 \quad B)(1+B)(1 \quad i B)(1+i B)$. Hence, an application of the $\Delta_{4}$ filter assumes the presence of the non-seasonal unit root 1 and of the seasonal unit roots -1 and $\pm i$. The HEGY method allows one to find, for example, the roots $\pm i$, and thus the application of the $\left(1+B^{2}\right)$ filter to establish stationarity. The auxiliary regression for the HEGY approach is

$$
\Delta_{4} X_{t}=\mu+\pi_{1} Z_{1, t-1}+\pi_{2} Z_{2, t-1}+\pi_{3} Z_{3, t-2}+\pi_{4} Z_{3, t-1}+\sum_{i=1}^{n} \phi_{i} \Delta_{4} X_{t-i}+u_{t},
$$

where $\mu$ can include a constant, seasonal dummies and trend, and where the values of $Z_{i}$ are defined by $Z_{1 t}=\left(1+B+B^{2}+B^{3}\right) X_{t}, Z_{2 t}=\left(-1+B-B^{2}+B^{3}\right) X_{t}$ and $Z_{3 t}=\left(-1+B^{2}\right) X_{t}$. When all $\pi_{i}$ are zero, the process $X_{t}$ is $I(0,1)$, and when they are all unequal to 0 , the process is stationary. Evidence has been found for the root 1 when $\pi_{1}$ is 0 , for the root -1 when $\pi_{2}=0$ and for the roots $\pm i$ when $\pi_{3}=\pi_{4}=0$. See HEGY for the details and also for the critical values of the $t$ statistics for $\pi_{1}$ and $\pi_{2}$ and for the $F$ statistic for the hypothesis $\pi_{3}=\pi_{4}=0$. In Franses (1991a) the HEGY approach is applied to monthly data and the conclusions show that the assumption of too many roots can have a considerable impact on forecasting.

A common characteristic of the auxiliary regressions in (1) and (2) is that the parameters are assumed to be constant over the seasons. A periodic approach that allows the parameters to vary assumes that $X_{1}$ can be modelled as a periodic autoregressive process [PAR] of order $p$ [see Franses (1993) for more details]. The process can be expressed as 


$$
X_{t}=\sum_{s=1}^{4} \alpha_{s} D_{s t}+\sum_{j=1}^{p} \sum_{s=1}^{4} \phi_{j s} D_{s t} X_{t-j}+\varepsilon_{t},
$$

where the autoregressive parameters vary over the seasons. If we decompose the values of $X_{t}$ into four subseries, say $x_{s T}$, which are the annual series of the observations per quarter, and stack these values of $x_{s T}$ into a vector of quarters [VQ], say $x_{T}, x_{T}$ can be modelled as

$$
A_{0} x_{T}=\mu+A_{1} x_{T-1}+\cdots A_{m} x_{T-m}+\varepsilon_{T}
$$

In the above equation the value of $m$ depends on the $p$ in (3), $A_{0}$ is a lower triangular matrix, and the values of $\mu$ and $\varepsilon_{T}$ are $(4 \times 1)$ vectors of means and errors, respectively, and the values of $A_{i}$ are $(4 \times 4)$ parameter matrices. For example, a $\operatorname{PAR}(1)$ can be written as (4) with

$$
A_{11}=\left[\begin{array}{cccc}
1 & 0 & 0 & 0 \\
-\phi_{2} & 1 & 0 & 0 \\
0 & -\phi_{3} & 1 & 0 \\
0 & 0 & -\phi_{4} & 1
\end{array}\right] \text { and } A_{1}=\left[\begin{array}{llll}
0 & 0 & 0 & \phi_{1} \\
0 & 0 & 0 & 0 \\
0 & 0 & 0 & 0 \\
0 & 0 & 0 & 0
\end{array}\right]
$$

It is assumed that $\varepsilon_{T} \sim N_{4}\left(0, \sigma^{2} I\right)$. Note that the index $T$ runs from 1 to $n / 4$.

For many economic time series, including those that will be discussed in section 4 , it applies that

$$
A_{0} x_{T}=\mu+A_{1} x_{T-1}+\varepsilon_{T}
$$

is an appropriate model. Rewriting this model into

$$
x_{T}=A_{0}^{-1} A_{1} x_{T-1}+\tau+\omega_{T},
$$

where $\omega_{T}=A_{0}^{-1} \varepsilon_{T}$ and $\tau=A_{0}^{-1} \mu$, one obtains a vector autoregressive process of order 1 for $x_{T}$.

The VQ method to test for seasonal unit roots amounts to applying the Johansen and Juselius (1990) cointegration method to the model in (6) or to checking whether (6) can be rewritten as

$$
\begin{aligned}
\Delta_{1} x_{T} & =\left(A_{01}^{-1} A_{1}-I\right) x_{T-1}+\tau+\omega_{T} \\
& =\alpha \beta^{\prime} x_{T-1}+\tau+\omega_{T},
\end{aligned}
$$

where $\alpha$ and $\beta$ are $(4 \times r)$ matrices with $0<r<4$ and where $r$ is the number of cointegration vectors. The matrix $\beta$ represents the cointegration space. The $\alpha$ parameters reflect the dynamic adjustment to disequilibrium errors. Note that the $\Delta_{1}$ filter for $x_{T}$ corresponds to the $\Delta_{\downarrow}$ filter for $X_{t}$.

It can be recognized that this $\Delta_{4}$ filter for $X_{t}$ assumes that there are no cointegration relations between the elements $x_{s T}$ and that if $x_{1}$ is stationary, the elements $x_{s T}$ are stationary. Hence, $\Delta_{4}$ implies that $r=0$, and stationarity of the $x_{s T}$ implies that $r=4$. Intermediate cases are established by finding one, two or three cointegration relations. Suppose one detects three such relations, and suppose that the cointegration space $\beta$ can be spanned by the vectors $(1,-1,0,0)^{\prime},(0,1,-1,0)^{\prime}$ and $(0,0,1,-1)^{\prime}$. In this case one has found evidence for the need of the $\Delta_{1}$ filter. This is because these restrictions imply that the variables $x_{4 T}-x_{3 T}, x_{3 T}-x_{2 T}$ and $x_{2 T}-x_{1 T}$ are stationary. This assumption can be tested with the techniques developed in Johansen and Juselius (1990). Similarly, restrictions like $(1,+1)$, in the case of three cointegration relations, refer to the presence of the seasonal unit root -1 since $(1,+1)$ corresponds to the $(1+B)$ filter. When two cointegration relations appear to be present and it holds true that $x_{4 T}-x_{2 T}$ and $x_{3 T}-x_{1 T}$ are stationary, then one can apply the $\left(1-B^{2}\right)$ filter. Extension to other combinations of unit roots is straightforward.

It can thus be seen that the VQ method generalizes the HEGY approach, since it allows testing for (non-)seasonal unit roots in a periodic model. When the $x_{s T}$ series in (6) are replaced by $\Delta_{1} x_{s T}$, it can be 
shown that the VQ method can also generalize the OCSB method. In case all restrictions related to (seasonal) unit roots are rejected, one can consider a univariate periodic error correction model (PECM), denoted as in (7), or more conveniently as

$$
A_{0} \Delta_{1} x_{T}=\left(A_{0} \alpha\right) \beta^{\prime} x_{T-1}+\mu+\varepsilon_{T}
$$

where $\beta^{\prime} x_{T-1}$ represents the error correcting variables and where the error term $\varepsilon_{T}$ is a vector white noise process in contrast to $\omega_{T}$. Note that the adjustment parameters $A_{0} \alpha$ are not necessarily negative.

\section{A Monte Carlo study}

To evaluate the effects of periodic and non-periodic processes on the test results of the HEGY, OCSB and VQ approaches, we carried out a limited simulation experiment that focuses on the differences between the methods. For illustrative purposes, we consider the autoregressive model of order 1 for $X_{t}$, with a possibility of periodically varying parameters. Extensions to higher order models and to models for $\Delta_{1} X_{t}$ and $\Delta_{4} X_{t}$, i.e. $I(1,0)$ and $I(0,1)$ processes, yield similar conclusions with respect to the effect of periodicity.

Denoting $\varepsilon_{t}$ as the vector of observations independently drawn from the standard $N(0,1)$ distribution, the data generating processes (DGPs) are

Table 1

Testing for non-stationary (seasonal) behaviour with three different methods. The cells show the frequency of selecting an order of integration

\begin{tabular}{|c|c|c|c|c|}
\hline \multirow[t]{2}{*}{$\mathrm{DGP}^{a}$} & \multirow[t]{2}{*}{ Method" } & \multicolumn{3}{|c|}{ Order of integration ${ }^{\mathrm{c}}$} \\
\hline & & $I(0,0)$ & $I(1.0)$ & $I(0,1)$ \\
\hline \multirow{3}{*}{ (i) } & OCsB & 0.937 & 0.061 & 0.002 \\
\hline & HEGY & 0.802 & 0.146 & 0.006 \\
\hline & VQ & 0.794 & 0.061 & 0.001 \\
\hline \multirow[t]{3}{*}{ (ii) } & OCSB & 0.088 & 0.912 & 0.000 \\
\hline & HEGY & 0.121 & 0.820 & 0.007 \\
\hline & VQ & 0.258 & 0.379 & 0.002 \\
\hline \multirow[t]{3}{*}{ (iii) } & OCSB & 0.949 & 0.048 & 0.003 \\
\hline & HEGY & 0.758 & 0.187 & 0.009 \\
\hline & $\mathrm{VQ}$ & 0.773 & 0.030 & 0.000 \\
\hline \multirow[t]{3}{*}{ (iv) } & OCsB & 0.967 & 0,015 & 0.018 \\
\hline & HEGY & 0.812 & 0,133 & 0.011 \\
\hline & VQ & 0.833 & 0.023 & 0.001 \\
\hline \multirow[t]{3}{*}{ (v) } & OCSB & 0.173 & 0.722 & 0.047 \\
\hline & HEGY & 0.026 & 0.753 & 0.159 \\
\hline & VQ & 0.067 & 0.032 & 0.000 \\
\hline
\end{tabular}

The number of replications is 5000 . The data-generating processes can be found in Eqs. (5)-(9). The $\varepsilon$, values for these DGPs are equal.

" The methods OCSB, HEGY and VQ are discussed in the text around Eqs. (1). (2) and (3), respectively. In this case, the HEGY and VQ method do not incorporate the $1(1,1)$ process.

- The numbers in the cells do not necessarily add to 1 , e.g. one can also find the presence of root -1 in the HEGY method, and this does not translate into the $I(d, D)$ notation. Furthermore, the number of times the OCSB method opts for the $I(1,1)$ order is not reported. The test outcomes are based on a $5 \%$ significance level for the OCSB an HEGY methods, and on a $10 \%$ level for the VO method. This is because the OCSB and HEGY methods use several tests at a $5 \%$ level. The overall significance level is then also about $10 \%$. The VQ model is of order 1 . The number of lags in the test equations for the HEGY and OCSB methods are set equal to that number $p, p=5, \ldots, 0$, for which there appeared to be no significant autocorrelation. 


$$
\begin{aligned}
& X_{t}=0.5 X_{t-1}+\varepsilon_{t}, \\
& X_{t}=0.9 X_{t-1}+\varepsilon_{t}, \\
& X_{t}=0.5 X_{t-1}+\psi_{s}^{1 / 2} \varepsilon_{t}, \quad \text { with } \psi_{1}=1.25, \psi_{2}=0.8, \psi_{3}=0.5, \psi_{4}=2.0, \\
& X_{t}=\phi_{x} X_{t-1}+\varepsilon_{t}, \text { with } \phi_{1}=0.2, \phi_{2}=0.4, \phi_{3}=0.6, \phi_{4}=0.8
\end{aligned}
$$

and

$$
X_{t}=\phi_{s} X_{t-1}+\varepsilon_{t}, \quad \text { with } \phi_{1}=1.25, \phi_{2}=0.8, \phi_{3}=0.9, \phi_{4}=1.11
$$

The index $s$ indicates that the parameter varies over the seasons. The fifth process is a periodically integrated autoregressive model of order 1 [see Osborn and Smith (1989)]. This is because the product of the $\phi$ parameters equals 1, i.e. $\phi_{1} \phi_{2} \phi_{3} \phi_{4}=1$, but not all $\phi_{s}$ are equal to 1 . A property of model (13), with respect to the VQ approach in (7), is that there are three cointegration relations between the elements $x_{s T}$ and that the restrictions $(1,-1)$ are not valid. Therefore this process is not of the $I(d, D)$ type.

The results in Table 1 show the frequencies of the selection of a filter $I(d, D)$, where $d$ and $D$ are either 0 or 1 . The true order is $I(0,0)$ in the cases of (i) through (iv), and it is clear that, with respect to detecting this order, the OCSB method outperforms the other methods for the DGPs (i), (iii) and (iv). The VQ method seems to be preferable for (ii), although the method still does not work well in nearly $75 \%$ of the cases. Moreover, note that for (ii) the VQ approach is likely to indicate that $X_{t}$ is periodically integrated. Furthermore, it can be seen that changing variances do not dramatically affect the performance of either of the tests and that the OCSB method yields rather stable outcomes for (i), (iii) and (iv).

The effect of a periodic process that is non-stationary, i.e. the model in (v), is displayed in the last rows of Table 1 . In about $70 \%$ of the cases, the OCSB and HEGY methods indicate incorrectly that the time series is $I(1,0)$. On the other hand, the VQ approach finds an $I(d, D)$ model in only $10 \%$ of the cases, leaving the remaining $90 \%$ to a periodically integrated model.

\section{UK macroeconomic variables}

A survey of seasonal patterns in UK macroeconomic time series is given in Osborn (1990). The methods used are the HEGY and the OCSB methods. For five of these series it is concluded that the series is $I(2,0)$, i.e. requires the use of the $(1-B)^{2}$ filter. In Franses $(1991 b)$ it is argued that the need for this filter may be established by one of the properties of the HEGY method, that is when the true DGP is a stationary AR(1) process, it seldom rejects the presence of the unit root 1 . This is because the autocorrelations of the $Z_{1 t}$ process [see below (2)] are of higher values than those of the original AR(1) process. Hence, in this section it is assumed that the order of integration is at most $I(0,1)$.

In Table 2, the results for $25 \mathrm{UK}$ macroeconomic time series are displayed. The definitions and sample sizes of the series can be found in the appendix to Osborn (1990). The table only reports the results of the VQ and HEGY methods, since the VQ method is a periodic generalization of the HEGY approach. The HEGY outcomes are taken from Table 2 in Osborn (1990). The order of the VQ model in (4) is set equal to 1 , since preliminary analysis, i.e. the estimation of $\operatorname{PAR}(p)$ processes like (3), indicated that only the parameter matrices $A_{0}$ and $A_{1}$ contain non-zero elements.

Several conclusions can now be drawn from Table 2. The first is that, as far as the VQ method is concerned, none of these UK series appears to be of order $I(0,1)$, since $r=0$ is not detected. This implies that the use of the $\Delta_{4}$ filter for these time series would result in overdifferencing. The second conclusion is that, when the VQ approach indicates that a series is $I(1,0)$, in most instances so also does the HEGY method. For example, a series like stock prices, which one would expect to be a $\Delta_{1}$ series, is found to be so by both methods. The third conclusion to be drawn is that the seasonal unit roots -1 and 
Table 2

Evidence of seasonality in UK macrocconomic variables

\begin{tabular}{|c|c|c|c|c|}
\hline \multirow[t]{2}{*}{ Variable } & \multicolumn{2}{|l|}{ HEGY' } & \multicolumn{2}{|c|}{$\mathrm{VQ}^{\mathrm{b}}$} \\
\hline & Roots & Filter & $r$ & Integration \\
\hline GDP & $\pm 1, \pm i$ & $\left(1-B^{4}\right)$ & 2 & $\mathrm{Pl}$ \\
\hline Consumption & $\pm 1, \pm i$ & $\left(1-B^{4}\right)$ & 2 & PI \\
\hline Durables & $\pm 1, \pm i$ & $\left(1-B^{+}\right)$ & 2 & PI \\
\hline Non-durables & $\pm 1, \pm i$ & $\left(1-B^{4}\right)$ & 2 & $\mathrm{PI}$ \\
\hline $\begin{array}{l}\text { Government } \\
\text { consumption }\end{array}$ & \pm 1 & $\left(1-B^{2}\right)$ & 1 & PI \\
\hline Investment & 1 & $(1-B)$ & 1 & PI \\
\hline Private & 1 & $(1-B)$ & 2 & PI \\
\hline Public & $\pm 1, \pm i$ & $\left(1-B^{4}\right)$ & 1 & $\mathrm{PI}$ \\
\hline Stockbuilding & -1 & $(1+B)$ & 4 & stationary \\
\hline Irade balance & 1 & $(1-B)$ & 3 & $(1-B)$ \\
\hline Exports & 1 & $(1-B)$ & 3 & PI \\
\hline Imports & 1 & $(1-B)$ & 2 & $\mathrm{PI}$ \\
\hline $\begin{array}{l}\text { Factor } \\
\text { adjustment }\end{array}$ & 1 & $(1-B)$ & 2 & PI \\
\hline Real income & 1 & $(1-R)$ & 2 & PI \\
\hline Employment & 1 & $(1-B)$ & 3 & $(1-B)$ \\
\hline Vacancies & - & - & 4 & stationary \\
\hline Workforce & 1 & $\left(\begin{array}{ll}1 & B\end{array}\right)$ & 2 & PI \\
\hline Productivity & $\pm 1, \pm i$ & $\left(1-B^{4}\right)$ & 2 & PI \\
\hline Earnings & 1 & $(1-B)$ & 3 & $(1-B)$ \\
\hline Prices & 1,1 & $(1-B)^{2}$ & 3 & $(1-B)$ \\
\hline Stock prices & 1 & $(1-B)$ & 3 & $(1-B)$ \\
\hline M0 & 1 & $(1-B)$ & 3 & $\mathrm{PI}$ \\
\hline M4 & 1,1 & $(1-B)^{2}$ & 3 & $\mathrm{PI}$ \\
\hline Interest rate & 1 & $(1-B)$ & 3 & $\mathrm{PI}$ \\
\hline Exchange rate & \pm 1 & $\left(1-B^{2}\right)$ & 1 & PI \\
\hline
\end{tabular}

"The HEGY method is described in the text around Eq. (2). A - means that no filter has to be applied, since the variable is found to be stationary.

'The VQ method is described around Eq. (7). PI means that the time series is periodically integrated. The significance level for the test on the value of $r$ is $20 \%$, while that of the test for parameter restrictions in the cointegration vectors is $1 \%$.

$\pm i$ are not found with the VQ method. Instead, it appears that many time series are periodically integrated and that a univariate periodic error correction model can be more appropriate. This implies that, for these series, non-stationarity and seasonality cannot be separated. Finally, in cases of periodic integration, the HEGY method concludes in almost all instances that the time series is either $I(0,1)$ or $I(1,0)$. These findings support the simulation results in Table 1.

\section{Implications for modelling and forecasting}

The outcome discussed in the previous section, that many UK macroeconomic time series are periodically integrated, can have implications for modelling and forecasting. We illustrate these implications by means of two examples. With respect to the following empirical estimation results, we note that all models are checked for residual autocorrelation of orders 1 and 4 (with $F$-type Lagrange multiplier tests), for $\mathrm{ARCH}(1)$ and $\mathrm{ARCH}(4)$ (with $\chi^{2}$ tests), and for normality of the residuals. These diagnostic results are not shown for reasons of space. Furthermore, the final models have been found after a general-to-simple specification search, and the diagnostic checks do not indicate misspecification.

First, consider the log import series, to be denoted as $i m_{t}$. An adequate model for this series, for $1956.1-1988.4$, is 


$$
i m_{t}=\hat{\mu}_{s}+\hat{\phi}_{s} i m_{t-1}+\hat{\varepsilon}_{l}, \quad s=1,2,3,4
$$

with

$$
\begin{array}{rrrr}
\hat{\mu}_{1}=0.341, & \hat{\mu}_{2}=-0.236, & \hat{\mu}_{3}=0.167, & \hat{\mu}_{4}=-0.270 \\
(0.160) & (0.165) & (0.160) & (0.162) \\
\hat{\phi}_{1}=0.966, & \hat{\phi}_{2}=1.028 & \hat{\phi}_{3}=0.984, & \hat{\phi}_{4}=1.026 . \\
(0.017) & (0.017) & (0.017) & (0.017)
\end{array}
$$

The product $\hat{\phi}_{1} \hat{\phi}_{2} \hat{\phi}_{3} \hat{\phi}_{4}$ equals 1.003 , and the $F$-type test for the hypothesis that this product is equal to 1 takes a value of 0.008 . An $F$-type test for the hypothesis that all $\phi_{s}$ are equal, i.e. that the model is non-periodic, yields a value of 3.433. Hence, the model in (14) is a nice example of a periodically integrated model, and Osborn and Smith (1989) show that it can outperform rival non-periodic models with respect to forecasting performance.

Model (14) is, however, not valid for all periodically integrated series in Table 2 . Some macroeconomic series are best described by a univariate periodic error correction model, as in (8). For example, consider the $\log$ of the durable consumption series for which we find that there are two cointegration relations in the annual series containing the quarterly observations. A search for simple models does not yield satisfactory results, hence we decide to fit a fully specified PECM to this series. Since we want to evaluate its forecasts, we restrict the sample to 1955.1-1985.4, and use the period 1986.1-1988.4 for evaluation. The two equilibrium relations between the $x_{s, T}$ values are given in Fig. 1 .

It is clear that they seem to be stationary. The two cointegration relations, $F E C_{T}$ and $S E C_{T}$, are

$$
F E C_{T}=0.740 x_{1 T}-0.215 x_{2 T}-0.633 x_{3 T}+0.071 x_{4 T}
$$

and

$$
S E C_{T}=0.027 x_{1 T}+0.447 x_{2 T}-0.778 x_{3 T}+0.441 x_{4 T} .
$$

Estimation results for the PECM in (8) are

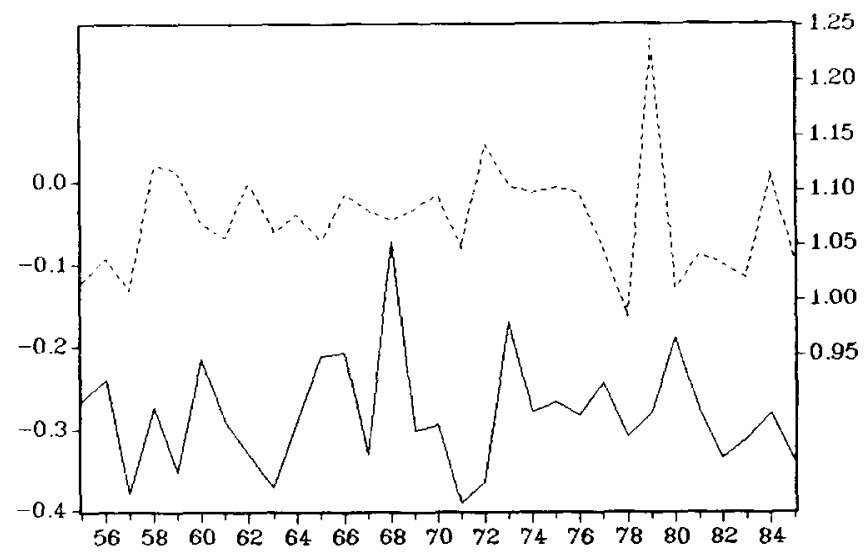

FEC -... SEC

Fig. 1. Two cointegration relations between the quarterly series $x_{s, \tau}$. 


$$
\begin{aligned}
\Delta_{1} x_{1 T}= & -0.458-1.804 F E C_{T-1}+\varepsilon_{1 T}, \\
& (0.060)(0.208) \\
\Delta_{1} x_{2 T}= & 1.647+0324 \Delta_{1} x_{1 T}-1.520 S E C_{T-1}+\varepsilon_{2 T}, \\
& (0.382)(0.124) \quad(0.360) \\
\Delta_{1} x_{3 T}= & -1.215+0.543 \Delta_{1} x_{2 T}+1.168 S E C_{T-1}+\varepsilon_{3 T} \\
& (0.429)(0.160)
\end{aligned}
$$

and

$$
\begin{gathered}
\Delta_{1} x_{4 T}=1.185+0.878 \Delta_{1} x_{3 T}-1.117 S E C_{T-1}+\varepsilon_{4 T} \\
(0.326)(0.146)
\end{gathered}
$$

Given that cointegration between time series also means that the forecasts will not diverge, it may be interesting to evaluate the forecasting performance of (15). As a rival non-periodic model, we have chosen a model with the time series transformed with a $\Delta_{4}$ filter, as implied by the outcome of the HEGY method. An adequate model for this $\Delta_{4} X_{t}$ series is

$$
\begin{gathered}
\Delta_{4} X_{t}=0.019+0.628 \Delta_{4} X_{t}{ }_{1}+\varepsilon_{t}-0.655 \varepsilon_{t}{ }_{4} \\
(0.009)(0.072)
\end{gathered}
$$

Also, the diagnostic checks do not reveal any misspecification for this model. From both estimated models (15) and (16), out-of-sample forecasts for 1986.1-1988.4 are generated for the level of the series $X_{t}$. The outcomes of forecast evaluation criteria are shown in Table 3 , where it is clear that the univariate PECM outperforms the rival non-periodic model. These results conform with those given in Osborn and Smith (1989).

However, it may be difficult to give economic meaning to the two equilibrium relations $F E C_{t}$ and $S E C_{t}$. Technically speaking it is difficult to give an interpretation to the cointegration vectors obtained from the Johansen and Juselius (1990) procedure without some kind of normalization. However, one aspect of a univariate PECM can be relevant to many economic time series, namely that the

\begin{tabular}{|c|c|c|}
\hline \multirow[t]{2}{*}{ Criteria $^{b}$} & \multicolumn{2}{|l|}{ Models $^{\mathrm{a}}$} \\
\hline & Non-periodic & PECM \\
\hline $\mathrm{ME}$ & 0.061 & 0.009 \\
\hline MAE & 0.066 & 0.056 \\
\hline MAPE & 0.759 & 0.642 \\
\hline $\operatorname{Max} A E$ & 0.170 & 0.121 \\
\hline RMSE & 0.079 & 0.067 \\
\hline Sign & 10 & 8 \\
\hline
\end{tabular}
disequilibrium errors have different impacts in different seasons. Hence, the effects of shocks on the pattern of $X_{t}$ values vary over the seasons. For time series that show cyclical patterns, such varying effects can cause the series to show asymmetric cyclical behaviour.

Univariate time series analysis is, of course, most often used as a data description tool, since univariate variables usually are part of a multivariate economic system. Therefore it is useful when there are many series to be periodically integrated to find out which type of multivariate models can generate univariate models like (14) and (15). When the equilibrium relations between the quarters of

Table 3

Forecasting performance of a non-periodic and a PECM for durable consumption

a The expressions of the models can be found in (16) and (15).

${ }^{b}$ The criteria are mean error (ME), mean absolute error (MAE), mean absolute percentage error (MAPE), the maximum values of the absolute errors (MaxAE), the root mean squared error (RMSE) and the number of times out of 12 forecasts the true observations exceed the forecasted ones (sign). 
the univariate series itself are replaced by cointegration relations between several series, one can extend the univariate PECM to a multivariate PECM or to a periodic cointegration modcl. This model extends the usual cointegration concept by allowing dynamic adjustment, as well as equilibrium relations, to vary over the seasons. A simple example of a single-equation periodic cointegration model is

$$
\Delta_{4} X_{t}=\alpha_{s}\left(X_{t-4}-\beta_{s} Y_{t-4}\right)+\varphi_{t}
$$

where $\alpha_{s}$ and $\beta_{s}$ are seasonally varying parameters. Franses and Kloek (1991) give a detailed account of the representation, estimation and testing issues for such a periodic cointegration model. In summary, the outcome that many UK macroeconomic time series seem to be periodically integrated may be used as a first step in specifying periodic cointegration models.

\section{Concluding remarks}

The paper presents evidence on the seasonal patterns of several UK macroeconomic variables, additional to those reported in Osborn (1990). The method used is a test procedure for seasonal unit roots that allows parameters to vary over the seasons. Simulation results indicate that the methods developed in Osborn et al. (1988) and Hylleberg et al. (1990) favour the use of an inappropriate differencing filter when the data-generating process is periodically integrated. The application of a periodic generalization of one of the previous two approaches yields the result that many UK macroeconomic series are periodically integrated.

This outcome has several implications. First, the seasonal behaviour of quarterly time series can be more complicated than the non-periodic behaviour that is often assumed to be the case. Secondly, models with constant parameters are misspecified in cases of periodicity. Hence, an improvement in forecasting performance can be expected when, for example, periodically integrated models are used. Thirdly, the appropriateness of non-stationary periodic models implies that, in some seasons, exogenous shocks have different effects on the time series pattern than those in other seasons. This suggests that variables with asymmetric cyclical behaviour can be modelled with a univariate periodic error correction model, in which case equilibrium relations between the seasons exist. An extension of this univariate model is given by a periodic cointegration model, i.e. a cointegration model in which theparameters in the long-run relations are allowed to vary, as well as the adjustment parameters.

\section{Acknowledgements}

We wish to thank Denise Osborn for making available all the data series and David Hendry, Estela Bee Dagum, an Associate Editor and two anonymous referees for helpful suggestions.

\section{References}

Franses, P.H., 1991a, "Seasonality, nonstationarity and the forecasting of monthly time series", International Journal of Forecasting 7, 199-208.

Franses, P.H., 1991b, "Moving average filters and unit roots", Economics Letters 37, 399-403.

Franses, P.H., 1993, “A multivariate approach to modeling univariate seasonal time series", Econometric Institute Report 9101 , Journal of Econometrics, in press.

Franses, P.II. and T. Kloek, 1991, "A periodic cointegration model of consumption", Econometric Institute Report 9172 , Erasmus University, Rotterdam.

Hylleberg, S., R.F. Engle, C.W.J. Granger and B.S. Yoo, 1990, "Seasonal integration and cointegration," Journal of Econometrics 44, 215-238. 
Johansen, S. and K. Juselius, 1990, "Maximum likelihood estimation and inference on cointegration-with applications to the demand for money", Oxford Bulletin of Economic and Statistics 52, 169-210.

Osborn, D.R., 1990, "A survey of seasonality in UK macroeconomic variables", International Journal of Forecasting 6, $327-336$. Osborn, D.R. and J.P. Smith, 1989, "The performance of periodic autoregressive models in forecasting seasonal UK consumption", Journal of Business and Economic Statistics 7, 117-127.

Osborn, D.R., A.P.L. Chui, J.P. Smith and C.R. Birchenhall, 1988, "Seasonality and the order of integration for consumption", Oxford Bulletin of Economics and Statistics 50, 361-377.

Biographies: Philip Hans FRANSES is affiliated to the Econometric Institute of the Erasmus University, Rotterdam, as a fellow of the Royal Netherlands Academy of Arts and Sciences. His research interests include model selection and seasonality in time series.

Gerbert ROMIJN is a Ph.D. student in macroeconomics at the Erasmus University, Rotterdam, affiliated to the Tinbergen Institute. 